ing Invitrogen (Carlsbad, California), Novagen (Madison, Wisconsin), Roche Applied Science (Indianapolis, Indiana) and others.

Some companies are now developing high-throughput methods for purifying proteins that are not tagged. "Typically most scientists work with tagged proteins through the discovery process. However, it is possible to lose some information because of tag interference in the biological assays. Removing the tag means removing some of the question marks," says Lisa Bradbury, director of R\&D for proteomics and cell therapy at Pall Corporation of East Hills, New York.

\title{
Purification tool bag
}

Several companies sell reagents for every step of the purification process - from cell lysis, to affinity purification resins, to desalting and concentrating reagents - adapted to highthroughput protocols that use standard 96-well plates. "We were recognized for our protein affinity-purification line. Now we are configuring it into a format that is automationfriendly," says Craig Smith, vice-president of R\&D at Pierce Biotechnology of Rockford, Illinois (part of Fisher Scientific). Among the company's offerings are the SwellGel Discs, pellets of dehydrated support that can be distributed into filter plates. When the protein is

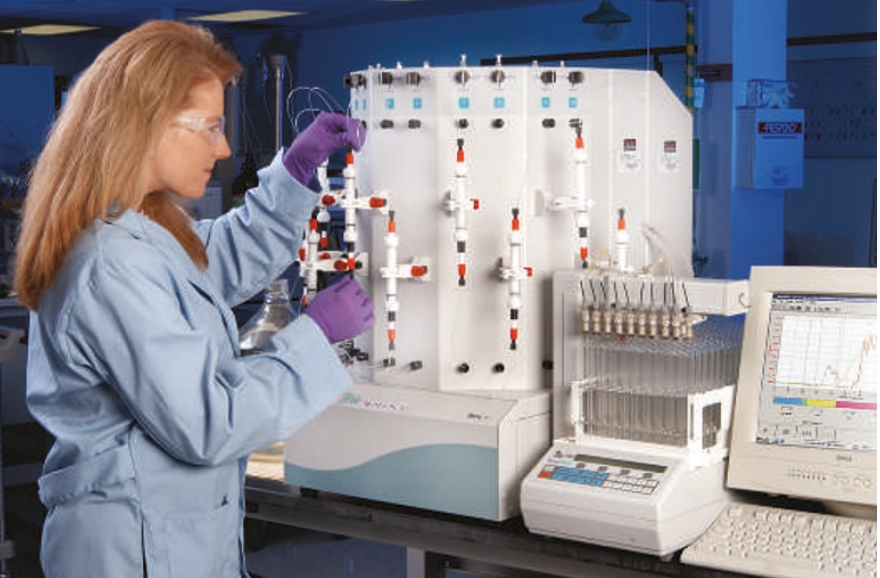

High-capacity purification: Teledyne Isoc's BioOptix 10.

added, the disc rehydrates to an affinity gel that binds tagged proteins. "We have taken out variability by having a dried pellet," says Smith. More recently, the company's Zeba Micro Desalt Spin Columns were configured to 96 well-plate format for desalting many small samples in parallel.

Pall Corporation also sells reagents for each step of the purification process. The AcroPrep 96 Filter Plate can be used for any type of chromatography, including immobilized metal affinity chromatography, in a multiwell platform. The format facilitates the optimization of various purification parameters, including the choice of metal ion and resin, the sampleto-resin ratio, and the elution conditions. Other reagents adapted to a high-throughput environment include the Mustang 96-well ion-exchange plates and Nanosep Centrifugal Devices.

QIAGEN, based in Hilden, Germay, offers the Superflow 96 BioRobot, a medium-scale purification kit for 6xHis-tagged proteins. "You load your pellets onto a robot, press a button, and you will have product in two hours," says Frank Schäfer, associate director for R\&D protein expression/proteomics. According to the company, purification can be done in denaturing or native conditions and yields $4 \mathrm{mg}$ of protein for each of 96 wells. "It is one system and one process for every sample," says Schäfer. QIAGEN sells a similar system that runs up to 24 samples in parallel to purify up to $30 \mathrm{mg}$ of protein for large-scale applications.

Novagen's host of reagents for high-throughput protein purification starts with the expression step. The Overnight Express Autoinduction Systems allow fully automated, parallel protein expression from E. coli cultures, and achieve high cell densities without monitoring growth or induction by the addition of isopropylbeta-D-thiogalactopyranoside. "The medium contains a blend of carbon sources optimized for tightly regulated, uninduced growth and automatic induction of protein expression," says Anthony Grabski, R\&D group leader for protein purification. The RoboPop Purification Kits contain reagents for the extraction and purifica-

\section{PROTEIN PURIFICATION FOR STRUCTURAL PROTEOMICS}

Structural genomics, or structural proteomics, aims to provide threedimensional information for all proteins. This information can be used to ascribe function to a protein and to reveal or invalidate drug targets. There are structural proteomics projects in both the public and private sectors around the world, including Germany, Japan and the United States.

In 2000, the US National Institute of General Medical Science in Bethesda, Maryland, launched an initiative to determine the structures of about 10,000 proteins representing different structural ('fold') families over the next decade. The studies will result in a public resource linking amino-acid sequence, structural and functional information, eventually allowing scientists to make the three-dimensional atomic structures of most proteins easily obtainable from their corresponding sequences.
One of several participating centres is the Genomics Institute of the Novartis Research Foundation in San Diego, a member of the Joint Center for Structural Genomics (JCSG). Scott Lesley (pictured below, left), who heads the institute's proteomics unit, says his group has processed 50,000 protein samples from more than 20,000 unique expression clones. The pipeline starts with a small screen protocol

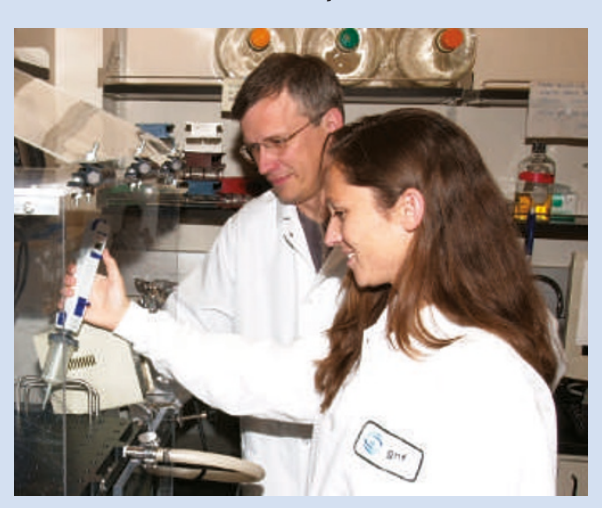

that uses samples in a 96-well format to evaluate how the targets behave at different steps in the procedure. "Those that are not behaving well after expression, purification or crystalization are set aside and then run through one of several salvage pathways," says Lesley. Such pathways include using different expression systems (vectors and cells), making point mutations in the CDNA to increase the likelihood it will be expressed, and trying different tags. "All the bacterial expression and purification is done in parallel and the majority is automated by custom instrumentation," says Lesley.

The targets that perform well are applied to a largescale purification protocol to yield
10-20 mg of protein. The JCSG has already deposited 239 structures in the protein database - mainly from the bacterium Thermotoga maritima, the mouse, and the yeast Saccharomyces cerevisiae representing at least 19 new fold families. "Most of the tools are in place, and we can increase both output and success rate by implementing salvage approaches in high throughput," says Lesley.

Similar efforts are underway in other countries. The Protein Structure Factory in Germany, an initiative of the German Human Genome Project and structural biologists from the Berlin area, targets human proteins for structure determination. "Our current focus is to determine the structures of particular protein-protein or protein-ligand complexes," says Christoph Scheich of the Max Planck Institute for Molecular Genetics in Berlin. 